\title{
Sur l'absence de théologie chez Shakespeare
}

\author{
Lachlan Mackinnon
}

\section{(2) OpenEdition \\ Journals}

\section{Édition électronique}

URL : http://journals.openedition.org/shakespeare/1147

DOI : 10.4000/shakespeare. 1147

ISSN : 2271-6424

Éditeur

Société Française Shakespeare

Édition imprimée

Date de publication : 1 novembre 1997

Pagination : 111-122

Référence électronique

Lachlan Mackinnon, "Sur l'absence de théologie chez Shakespeare ", Actes des congrès de la Société française Shakespeare [En ligne], 15 | 1997, mis en ligne le 01 janvier 2007, consulté le 29 avril 2019. URL : http://journals.openedition.org/shakespeare/1147 ; DOI : 10.4000/shakespeare.1147 


\section{S U R L' A B S E N C E D E T H É O L O G I E C H E Z S H A K E S P E A R E}

Dans A Midsummer Night's Dream, Theseus dit à Hippolyta que «The best in this kind are but shadows, and the worst are no worse if imagination amend them»(A Midsummer Night's Dream, V.1.210-11). Dans ces mots nous trouvons une sagesse attristée. Même la représentation la plus émouvante n'est qu'illusoire, et c'est seulement grâce aux spectateurs que tient l'illusion. En outre, l'imagination est capable de compenser les défauts de n'importe quelle représentation, si épouvantable qu'elle soit.

Le théâtre de Shakespeare est rempli de ces pensées tristes. Elles atteignent, peut-être, à leur niveau le plus exalté quand Prospero dit que :

These our actors,

As I foretold you, were all spirits, and

Are melted into air, into thin air ;

And like the baseless fabric of this vision,

The cloud-capped towers, the gorgeous palaces,

The solemn temples, the great globe itself,

Yea, all which it inherit, shall dissolve:

And, like this insubstantial pageant faded,

Leave not a rack behind.

(The Tempest, IV.1.148-56) ${ }^{1}$

A ce moment vertigineux, Prospero fusionne le monde et le théâtre. Celui-là est aussi irréel que celui-ci. Or, pour les spectateurs, il y a une signification de plus, parce que «the great globe itself» nomme non seulement 
notre planète mais aussi un théâtre. L'évanouissement du monde est égal aux vicissitudes du show-business.

Quand Jaques nous dit que «All the world's a stage» (As You Like It, II.7.139), il croit que la destination de la vie humaine est «mere oblivion», mais il ne suggère pas que les planches puissent se dissiper. Il y a eu une sentimentalité qui trouvait dans la renonciation de Prospero la voix de son créateur, un homme fatigué qui aurait été en train de se preparer enfin pour son retour au particulier. On ne l'entend plus de cette façon, mais le nihilisme de Prospero me semble décisif pour une compréhension de la métaphysique de Shakespeare.

Devant le tombeau à Stratford, il est impossible de ne pas se rendre compte du lien qui unit la vie de Shakespeare et celle de l'Église anglicane. Mais, il nous faut nous souvenir que nous ne connaissons rien des convictions de Shakespeare, ni de ses raisons pour vouloir rendre service à l'Église. Il est possible, par exemple, qu'un tel service ait signalé simplement le retour de la famille Shakespeare à la respectabilité publique. En même temps, les convictions catholiques se soutenaient souvent sous des formes protestantes. La situation même du tombeau ne nous dit rien de l'homme enterré sous la dalle.

Surtout, cet individu avait été homme de théâtre. Nous sommes convaincus, par exemple, que Henry IV sera un meilleur roi que Richard II simplement parce qu'il sait mieux se conduire en roi. Il jouera mieux le rôle, tandis que Richard ne l'a fait qu'en amateur. Richard s'est cru le représentant de Dieu en Angleterre, mais, après avoir été deposé, il entre dans une condition flottante. Pendant qu'il médite, il peut «play... in one person many people» (Richard II, V.5.31) :

Sometimes am I king;

Then treason makes me wish myself a beggar,

And so I am. Then crushing penury

Persuades me I was better when a king.

Then am I kinged again, and by and by

Think that I am unkinged by Bolingbroke,

And straight am nothing.

(Richard II, V.5.32-8)

Étourdi par la perte de son royaume, Richard a choisi une vie mentale où tout peut être renversé. Qu'il soit paysan ou roi, le rêveur éveillé s'amuse ici des délices de la souffrance. Il n'y a que le nom «Bolingbroke», qui résonne à travers ses pensées, pour nous rassurer qu'elles réfléchissent l'histoire qu'il a vécue. 
Comme on voit quand il fait cesser la contestation de Bolingbroke et de Mowbray, il est trop inconstant pour gouverner. Richard choisit donc le temps de l'inconstance, le temps cyclique qui est à la fois le temps du comédien, le temps de la comédie et le temps des rythmes biologiques ${ }^{2}$. De ce point de vue, on peut estimer plus exactement l'importance du jardinier et de ses hommes.

Quand ceux-ci se montrent, la Reine dit : «They will talk of state, for everyone doth so / Against a change»(III.4.28-9). Cela suppose que «everyone» - et avec quelle hauteur elle le dit - passe sous silence les affaires d'état sauf quand un changement menace. Ici, le discours est proprement horticole. «O, what pity is it», s'exclame le jardinier, «That he had not so trimmed and dressed his land / As we this garden !» (56-8).

Richard manque à son devoir de maintenir l'ordre dans son royaume, mais ce devoir est par implication ce que le bon Dieu a demandé à nos premiers aïeux. La déposition de Richard répète en écho la chute de l'homme.

Mais le conseil sage de Candide, qu'il nous faut «cultiver notre jardin», ne convient pas toujours aux princes. De par leurs fonctions, il leur faut vivre dans un temps non pas cyclique mais linéaire. Il leur faut aussi se soumettre à un mal qui est tout à fait différent du mal qui assiste à la vie cyclique.

Henry IV Part II nous montre cette différence d'une facon plus aiguë. Falstaff va accompagner son prince à la guerre, et il dit aux jeunes filles que «The underserver may sleep, when the man of action is called on» (II.4.3778). Pendant un moment, il se regarde en homme d'histoire. «Action» peut signifier simplement ce qui se passe sur la scène, mais le mot veut dire auss une bataille ou, notamment, un acte irréversible. Le sens du verbe anglais «to act» comprend, à peu près, celui des deux verbes français «jouer» et «agir». Ils se rencontrent naturellement dans le théâtre, et, chaque fois que Shakespeare se sert de «to act» et de ses formes, il nous faut décider jusqu'à quel point les deux sens se distinguent. Ici, plusieurs possibilités confrontent l'acteur. Aux extrêmes, Falstaff peut se montrer ou naïvement sincère ou ironique d'un air rusé. Cette liberté lui appartient parce que sa nature est à la fin comique.

La scène du départ de Falstaff précède une scène d'action historique, et puis nous nous trouvons dans le Gloucestershire. Mais nous ne le savons pas. Deux vieillards titubent sur la scène. «Come on, come on, come on !» (III.2.1) dit le premier, presque comme s'il était le metteur en scène. L'action du spectacle s'arrête pendant les cinquante et une lignes qui suivent, et on parle du passé. Le passé est peuplé d'individus nommés - «little John Doit of Staffordshire, and black George Barnes, and Francis Pickbone, and Will Squeal, a Cotswold man» (18-20), par exemple. Nous entendons les personnages d'un roman non écrit. Mais le passé se meurt. Comme Shallow le dit, «how many of my old acquaintance are dead» (33). Dans ces cinquante et une lignes, le mot «death» se prononce deux fois, et «dead» six fois. 
Silence. We shall all follow, cousin.

Shallow. Certain, 'tis certain; very sure,

very sure. Death, as the Psalmist saith, is

certain to all ; all shall die. How a good

yoke of bullocks at Stamford fair?

Shallow répétera que «Death is certain» (39), troublé par la mort de «old Double» (51), mais il semble qu'il n'ait aucune idée de l'outre-tombe. La mort arrive, simplement, aussi naturellement que les prix s'élèvent ou baissent au marché.

L'arrivée de Falstaff est l'arrivée de l'histoire dans le monde de Shallow. Ses essais de conscription sont peu honorables, mais ils occasionnent un éclair d'honneur au moment où Feeble cède à son destin :

Feeble. By my troth, I care not. A man can die but once. We owe God a death. I'll ne'er bear a base mind. An't be my destiny, so ; an't be not, so. No man's too good to serve's prince. And let it too good to serill he that dies this yeag is which way it will, he that dies this year is quit for the next.

Bardolph. Well said ; thou'rt a good fellow.

Feeble. Faith, I'll bear no base mind.

Pas exactement stoïque, Feeble évince un amour-propre légitime. Nous devons une mort au bon Dieu, mais le moment où elle sera exigée appartient au destin. Et, bien sûr, la mort règle tous les comptes. Néanmoins, Feeble ne se rendra jamais «base». Là où Shallow a semblé montrer par répétition une fixation à l'assurance de la mort, Feeble atteint à une espèce d'heroïsme. «I'll ne'er bear a base mind... I'll bear no base mind».

Feeble est capable de se raconter une histoire de lui-même qui ne se rapporte pas seulement au passé mais qui se projète dans l'avenir. Son «ne'er», son «jamais», nous dit qu'il s'est conçu en personnage et en homme de volonté. Ainsi devient-il homme d'histoire, si humble qu'il en soit.

Pour le jardinier, le mal est le désordre. Sous cette rubrique, nous pouvons parler de tous les maux de la chair, la mort naturelle y compris. Quand tout est bien ordonné, la vie du jardinier suit un cycle naturel. C'est son devoir de combattre le désordre qui menace toujours de dégrader le jardin, et, de son point de vue, le roi n'est qu'un jardinier à plus grande échelle. 
L'homme naturel, qui est aussi l'homme comique, doit donc éviter une mort trop immédiate. «Death is certain», mais il ne faut pas la chercher. Participer à l'histoire, c'est hasarder sa propre vie. Le temps linéaire de l'histoire ne permet aucun retour. Ce qui arrive n'arrive qu'une seule fois. Feeble quitte le monde de la comédie pour entrer dans celui de la tragédie. Il quitte un monde où le mal est dans l'ordre des choses et il entre dans un monde où le mal procède des faits et gestes des êtres humains.

Macbeth nous montre une transition comparable. Très attaché à sa femme, fier des honneurs que le roi lui a rendus, Macbeth est très aimable, mais il se fait assassin. Jusqu'à ce point, l'histoire n'est pour lui qu'une déformation professionnelle. Elle n'est pour lui que son devoir, et le choix n'est pas en cause. Sur le champ de bataille, il est fonctionnaire, et il est individu seulement chez lui. De haute naissance, il n'a pas pu se retirer entièrement de l'histoire, comme l'aurait pu Feeble, de naissance modeste, mais il lui a été néanmoins possible de conduire une vie essentiellement privée.

La question de la motivation de Macbeth est à la fois très importante et très difficile à résoudre. Le drame le fournit de trop de mobiles. En premier lieu, il y a les sorcières. Elles sont les premiers personnages qui apparaissent, et leur présence entoure d'une atmosphère mystérieuse tout ce qui suit. Ce qu'elles disent est toutefois peu convaincant. Leurs charmes sont assez terrifiants mais, par exemple, au moment où elles parlent de :

Scale of dragon, tooth of wolf,
Witches' mummy, maw and gulf
Of the ravined salt-sea shark,
Root of hemlock digged i'th' dark,
Liver of blaspheming Jew,
Gall of goat, and slips of yew
Slivered in the moon's eclipse,

(IV.1.22-8)

nous pouvons bien nous douter de la réalité des choses dont elles font mention. Il se peut bien que ces vieilles femmes ne soient sorcières que d'occasion et par hasard. Elles montrent les apparitions à Macbeth, mais pas avant que la série de meurtres se soit bien commencée. Les apparitions confirment qu'il a saisi une couronne stérile, que les descendants de Banquo en hériteront, et elles le prémunissent de façon ambiguë contre Macduff et contre la forêt. On veut souvent traiter les trois sorcières comme si elles étaient vraiment les Parques, mais le texte ne supporte point cette interprétation.

C'est-à-dire, sauf, les ajoutés où les sorcières rencontrent Hécate. Qui que ce soit qui les ait écrits a mal compris le texte de Shakespeare. Hécate blâme 
les sorcières :

Saucy and over-bold, how did you dare

To trade and traffic with Macbeth

In riddles and affairs of death?

Elle s'enrage parce que les vieilles ont eu affaire avec «a wayward son» (11) qui aime «for his own ends, not for you» (13). Elle se charge du destin de Macbeth et elle indique que les apparitions

Shall draw him on to his confusion

He shall spurn fate, scorn death, and bear

His hopes 'bove wisdom, grace, and fear ;

And you all know security

Is mortals' chiefest enemy. (29-33)

Tout le mystère du spectacle s'évanouit. C'est Hécate qui va détruire Macbeth ; ce que les apparitions auront à dire le conduira à sa mort. Avant de les voir, nous comprenons leur fonction et nous savons que Macbeth va mourir à cause de leurs promesses.

Ce manque de sagacité dramatique est assez étonnant pour rendre invraisemblable l'attribution de ces lignes à Thomas Middleton. L'inconnu qui les a écrites a mal compris le texte, mais le texte l'a troublé. Il a voulu donner aux supplices de Macbeth un sens métaphysique, mais il a réduit le héros au rôle de marionnette. Il a dégradé aussi les sorcières, qui deviennent agents du sort et sont privées de leur intérêt humain.

Bien que Lady Macbeth ait mis la virilité de Macbeth en question, et que les doutes qu'elle exprime le contraignent au moment de l'assassinat, il nous faut accepter enfin que la motivation de Macbeth procède de son for intérieur. Peu après avoir entendu qu'il sera roi, il se demande :

why do I yield to that suggestion Whose horrid image doth unfix my hair And make my seated heart knock at my ribs Against the use of nature ? Present fears Are less than horrible imaginings.

My thought, whose murder yet is but fantastical,

Shakes so my single state of man that function

Is smothered in surmise, and nothing is

But what is not. 
«Horrid» veut dire «horrible», mais aussi, par son étymologie latine, «bristling». L'image se hérisse comme ses cheveux. La fantaisie de Macbeth, plus horrible que ne puisse être aucune peur actuelle, lui propose un meurtre. Son «single state of man», soit sa condition solitaire, soit sa virilité isolée, soit son royaume à lui-même, tremble. L'humain, le sexuel et le politique se rencontrent ici avec une force effrayante. Macbeth est en train de découvrir la vérité de l'ambition.

C'est à Macbeth de choisir, et il choisit le mal. Je ne veux pas dire qu'il est l'homme des écrivains existentialistes, l'homme qui se choisit à chaque instant. La question à laquelle l'existentialisme n'a jamais su répondre, c'est celle de pourquoi on choisit une chose de préférence à une autre. Privé de son historicité et de toute valeur transcendentale, l'homme se trouve sans raison de faire quoi que ce soit. Jean-Paul Sartre s'est servi d'un raisonnemment presque kantien, qu'il fallait se lier à la cause du peuple, mais il est bien difficile de comprendre que cette opinion n'ait tiré aucun parti de son origine d'une préférence décidée auparavant. La division entre le pour-soi et l'en-soi est trop nette : il faut toujours que le pour-soi se soit incarné, et nos choix surgissent de la relation entre l'incarné et l'incarnation déterminée.

On pourrait donc dire que le mal vient à Macbeth de lui-même. Mais ce ne serait pas vrai. Le mal qui vient à Duncan, la mort, vient de Macbeth, mais les maux qui affligent Macbeth viennent de plusieurs origines. Il quitte le monde naturel, le monde comique de la domesticité, et en revanche les bons naturels se retirent de lui. Il ne dort plus. Le temps assume une forme linéaire grise :

Tomorrow, and tomorrow, and tomorrow Creeps in this petty pace from day to day To the last syllable of recorded time.

\section{(V.5.18-20)}

A l'annonce de la mort de sa femme, il a dit que «She should have died hereafter» (16). On a discuté longtemps pour savoir s'il a voulu dire qu'elle n'aurait pas dû mourir encore ou qu'elle aurait dû mourir plus tard, mais la question semble sans intérêt. Dans un temps dénué de sens, la mort d'aucun individu ne pèse sur lui. Comme s'il reconnaissait lui-même l'ambiguité de ce qu'il vient de dire, Macbeth exprime son ennui total.

Une fois la violence insérée dans la politique et elle y restera jusqu'à ce qu'une violence plus grande vienne la subordonner. Hannah Arendt, par exemple, y a toujours insisté. Nous savons par ses exploits que Macbeth est un homme d'une violence immodérée mais réglée. Tout va bien pendant que sa violence s'applique à maintenir l'ordre politique contre ses ennemis, mais quand elle entre dans le processus politique il faut l'en chasser - violemment. 
Quand les généraux saisissent le pouvoir, les colonels le leur prennent, puis les capitaines à leur tour, et on finit avec un carnage suivi d'une fatigue énorme. Ou, comme chez Macbeth, la violence se soutient par ses propres moyens jusqu'à ce qu'elle s'ennuie. C'est une nécessité, ici tragique.

Macbeth subit des maux de deux espèces. Les uns se présentent du temps cyclique, les autres de la nécessité publique. Les uns sont les incapacités qui accompagnent naturellement la trahison de sa propre nature, les autres sont les conséquences automatiques qu'occasionne une violation du monde politique. Il n'y a aucun besoin d'aller chercher dans la métaphysique pour comprendre le destin de Macbeth.

Aujourd'hui, nous courons le danger d'oublier qu'il y a pire encore que la mort. Les maux dont nous nous protégeons sont, pour la plupart, ceux de la biologie : nous nous inquiétons de notre santé, nous faisons du jogging, nous espérons ne jamais vieillir. La mort que nous craignons consiste simplement à cesser de vivre. Ce sont les valeurs du consommateur.

Dans son livre The Human Condition, peut-être le plus important de son œuvre, Hannah Arendt a divisé la vie humaine en trois parties ${ }^{3}$. Pour les deux premières, il y a la distinction française qu'elle a trouvée entre «travailler» et «ouvrer». Pour Arendt, le travailleur fait ce qu'il faut pour vivre. Le travail consiste à remplacer ce qu'on a mangé ou ce qui renouvelle le monde humain qui s'écroule. L'ouvrier, cependant, crée ce qui durera. Il est l'architecte, le poète, le peintre, le charpentier, le potier. Le travailleur est Homo Laborans, l'ouvrier Homo Faber. Pour Arendt, la troisième partie de la vie est la vie active. Ici, il s'agit de ce qui apparaît dans le monde publique, des actions ou des mots qui commencent quelque chose de nouveau. Ici, et seulement ici, ditelle, l'homme se découvrit.

Rapidement, on comprend que la vie du travailleur est la vie cyclique, convenable à la comédie, tandis que la vie active se trouve réfléchie dans la tragédie ou dans l'histoire.

Les genres dramatiques corroborent le dire d'Arendt. Chez Shakespeare, nous trouvons vite la vie du travailleur et la vie active, mais la vie de l'ouvrier est bien plus difficile à découvrir. On pourrait penser, par exemple, au Cinna de Julius Cassar. «Tear him for his bad verses, tear him for his bad verses» (III.3.30-31), s'écrie le Quatrième Plébéien, et Cinna disparaît avec la foule. Au moment où le Poète anonyme arrive pour plaider «Let me go in to see the generals» (IV.6.178), il dit en plus :

Love and be friends, as two such men should be,

For I have seen more years, I'm sure, than ye. (183-4)

La rime est assourdissante, mais pas pour les généraux, qui l'ont bien écoutée. 
Cas. Ha, ha! How vilely doth this cynic rhyme! Brut. Get you hence, sirrah; saucy fellow, hence! Cas. Bear with him, Brutus, 'tis his fashion.

Brut. I'll know his humour when he knows his time.

What should the wars do with these jigging fools?

Immédiatement, Cassius croit que le Poète est cynique. On peut se souvenir de l'affirmation de Paul Valéry, que les vers habiles sont l'art d'un sceptique profond. Brutus veut le chasser, Cassius prêche la tolérance, après quoi Brutus insiste que la poésie n'a pas de place en temps de guerre.

A l'égard de cette indifférence à la poésie, il faut considérer la seule occasion où un artiste achève un chef-d'œuvre sur la scène shakespearienne. La statue de Hermione que nous voyons dans The Winter's Tale «has motion in't» (V.3.676), contient du mouvement, mais elle se montre être la femme elle-même. L'art existe pour se dissoudre dans la vie. Dans Hamlet on oublie «The Murder of Gonzago» aussitôt que le spectacle a eu son effet voulu. Tant pis pour les tragédiens.

Il semble que Shakespeare ne se soit jamais troublé du destin de sa production littéraire. Les poèmes survivraient ou non; il avait eu quelque chose à faire avec leurs éditions mais on ne sait pas quoi. Sauf, peut-être, les Sonnets. Quant au théâtre, il apparaît que Shakespeare n'a rien fait pour s'assurer une postérité. Virgile et Franz Kafka ont voulu qu'on détruise leurs œuvres ; on ne l'a pas fait, heureusement, mais ce désir montre au moins leur avis. Les œuvres n'ont pas été perfectionnées. Nous ne connaissons rien de ce que Shakespeare ait pu penser de son théâtre. C'est comme si la vie de l'ouvrier lui était inconnue. Bien sûr, les Sonnets parlent de leur durée, mais il faut se souvenir que les Sonnets ne sont pas l'expression sincère du poète lui-même ${ }^{4}$. Plutôt, ils sont un jeu d'esprit, un examen du genre «sonnetsequence» ${ }^{5}$.

Cette indifférence à l'art est une indifférence à la dimension transcendentale. La vie du travailleur produit ce dont on a immédiatement besoin et ce que l'on consomme. La vie active trouve son but dans les actes ou dans les paroles qui ne s'oublieront jamais. La vie de l'ouvrier, cependant, se nie en faveur de ce qui durera sous une forme matérielle. Cette durée ne dépend ni de la volonté, ni de la mémoire, des générations suivantes. Soit table, soit poème, l'ouvrage dure, sans nécessité absolue de l'aide apportée par d'autres êtres humains. Il participe d'une dimension transcendentale.

(Il est vrai que la poésie orale a eu besoin du souvenir humain, mais il est aussi vrai qu'elle n'a été souvent elle-même qu'une forme du souvenir dédié à la vie active. Le poème comme nous le comprenons, à partir de 
Hésiode et de Homère, se fie à l'écriture et c'est ainsi qu'il gagne sa durée matérielle. Il est aussi vrai qu'à l'âge de Shakespeare les dramaturges ne faisaient pas imprimer leurs pièces. Il est néanmoins étonnant qu'un tel écrivain ait pu accepter de se rendre éphémère).

Si la dimension transcendentale manque au thêâtre de Shakespeare, il contient toujours des exemples d'une transcendence bornée. D'espèce, il y a la mort de Cleopatra. Voici une femme qui a été reine et amante de rois et d'empereurs. Maintenant, sa vie publique s'approche d'une crise. Son dernier amant s'est suicidé. Un jeune homme, un grand garçon, l'a vaincue. Ce dernier ne connaît que l'amour fraternel. En spectateurs, nous savons bien que l'histoire est de son parti. Cleopatra choisit la mort de préférence à la honte. Elle a peur d'être présentée comme un spectacle aux citoyens de Rome, «Mechanic slaves» (Antony and Cleopatra,V.2.205).

Charmian entre sur la scène, et Cleopatra ordonne :

Show me, my women, like a queen. Go fetch

My best attires. I am again for Cydnus

To meet Mark Antony. (223-5)

«Show me» : rendez-moi spectaculaire. Shakespeare nous a convaincus de sa splendeur à Cydnus par les mots de Enobarbus, le cynique impressionné. Maintenant, Cleopatra se rend triomphale.

Dolabella arrive. Il est pensif :

Cæsar, thy thoughts

Touch their effects in this. Thyself art coming

To see performed the dreaded act which thou

So sought'st to hinder. (324-7)

Son langage devient théâtral. Cleopatra a su mettre en scène sa propre mort. Quand Octavius regarde les corps de la reine et de ses femmes, il dit :

Bravest at the last,

She levelled at our purposes, and, being royal,

Took her own way. The manner of their deaths?

I do not see them bleed. (329-32)

Il lui faut avouer sa magnificence, mais il veut apprendre comment l'effet s'est produit. Il admire l'emploi de l'aspic parce que :

If they had swallowed poison, 'twould appear

By external swelling; but she looks like sleep,

As she would catch another Antony 
In her strong toil of grace. (339-42)

«Like sleep»: elle ne s'est pas simplement soumise à un processus naturel, elle l'est à peu près devenue. Cæsar sait déjà que «She hath pursued conclusions infinite / Of easy ways to die» (349-50), et le jeu de mots élisabéthain qui unissait la mort et la jouissance résonne. La mort de Cleopatra est une victoire sexuelle. Elle échappe à l'histoire par un retour à la nature.

Du point de vue de la nature, la mort n'est qu'une rentrée à la terre dont nous avons été créés. Il est donc approprié que la mort arrive ici par une toute petite blessure. Cleopatra est morte en comédienne en se retournant vers les cycles naturels, l'amour et la jouissance inclus. «The stroke of death is as a lover's pinch» (290). Même le mot «stroke» hésite entre «coup» et «caresse».

Morte en comédienne, Cleopatra est aussi morte en tragédienne. «I am fire and air ; my other elements / I give to baser life» (284-5). Pour que sa réputation soit glorieuse plutôt qu'honteuse, il lui faut mourir. Elle unit deux façons de vivre et deux genres dramatiques, à tel point que pendant quelques moments nous ne savons pas quel genre nous regardons.

Mais la transcendence reste théâtrale. L'histoire domine la fin de la scène avec le commandement de «High order in this great solemnity» (360). Au moment où Richard II se meurt, il dit à Exton, le meurtrier, que «thy fierce hand / Hath with the King's blood stained the King's own land» (Richard II, V.5.110). Enfin, il accepte encore son rôle historique, mais il n'a jamais pu entrer dans la vie comique, la vie des jardiniers, autant que l'a pu Cleopatra. Là où il est inconstant, elle est avisée. Son triomphe est un triomphe de la volonté.

La dimension de transcendance qui manque au théâtre de Shakespeare est enfin la dimension religieuse, la dimension de la théologie. Shakespeare nous montre tout ce qui se passe dans le temps, mais du temps où il n'y en aura plus il ne sait pas parler. Son humanisme est absolu. Pour lui, les maux sont ou comiques, ceux de la chair, ou tragiques, ceux de l'espoir déçu. Ils sont tellement liés aux genres dramatiques qu'à la question des origines du mal il ne saurait faire aucune réponse. 


\section{${ }^{\mathbf{N}}$ O T E S}

${ }^{1}$ Toutes les citations shakespeariennes ont été tirées de William Shakespeare, The Complete Works : Compact Edition, eds. Stanley Wells, Gary Taylor, John Jowett and William Montgomery (Oxford : Clarendon Press, 1988).

${ }^{2}$ Cf. John Bayley, Shakespeare and Tragedy (London, Boston, Melbourne and Henley : Routledge and Kegan Paul, 1981), p. 196, et Peter Conrad, The Everyman History of English Literature (London and Melbourne : J.M. Dent and Sons, 1985), p. 153

${ }^{3}$ Hannah Arendt, The Human Condition (Chicago and London : University of Chicago Press, 1958).

${ }^{4} \mathrm{Je}$ suis reconnaissant à $\mathrm{M}$. Ellrodt, qui m'a indiqué, en posant une question, que ce que j'avais écrit ici n'avait pas été exprimé en termes clairs. J'espère que $j$ 'ai trouvé une meilleure expression.

${ }^{5}$ Cf. Conrad,op. cit., p. 103-7. 